\title{
Condensational theory of stationary tornadoes
}

\author{
A. M. Makarieva, V. G. Gorshkov, and A. V. Nefiodor* \\ Theoretical Physics Division, Petersburg Nuclear Physics Institute, 188300 Gatchina, St. Petersburg, Russia
}

\begin{abstract}
Using the Bernoulli integral for air streamline with condensing water vapor a stationary axisymmetric tornado circulation is described. The obtained profiles of vertical, radial and tangential velocities are in agreement with observations for the Mulhall tornado, world's largest on record and longest-lived among the three tornadoes for which 3D velocity data are available. Maximum possible vortex velocities are estimated.
\end{abstract}

PACS numbers: 47.10.-g, 47.90.+a, 47.45.-n, 51.90.+r

\section{THE CONDENSATIONAL PRESSURE POTENTIAL}

Tornado circulation induced by water vapor condensation can be described as follows. Condensation of water vapor in the adiabatically ascending air results in a drop of air pressure by $\Delta p=p_{v}$, where $p_{v}$ is water vapor pressure at the Earth's surface. The decrease of pressure along the vertical axis sustains the ascending air motion with vertical velocity $w$ and induces a compensating horizontal air inflow with radial velocity $u$. The converging radial flow has maximal velocity at the surface, where the magnitude of the condensation-induced pressure drop is the largest. Radial velocity approaches zero at a certain height $z=h$, which approximately coincides with the cloud height. In the upper atmosphere at $z>h$ the condensed water is transported away from the condensation area by the strong updraft and outgoing air flow. It precipitates at a considerable distance from the center of the condensation area.

The continuity equation in the cylindrical system of coordinates relates radial $u$ and vertical $w$ velocities of the axially symmetrical vortex as $w=(h / r)(\partial u r / \partial r)$. The vertical and horizontal pressure gradient forces induced by condensation are $\Delta p / h$ and $\partial p / \partial r$, respectively. Equating the power of the vertical and radial air flow, $u \partial p / \partial r=w(\Delta p / h)$, and accounting for the continuity equation, we obtain $\partial p / \partial r=$ $\Delta p(u r)^{-1}(\partial u r / \partial r)$. This corresponds to pressure potential $p=\Delta p \ln u r+$ const [1]. Its exact derivation is given in work [2].

\section{THE BERNOULLI INTEGRAL FOR CONDENSATION-INDUCED TORNADOES}

For the high wind velocities of intense vortices to arise, the condensational pressure gradients within both tornadoes and hurricanes must significantly exceed turbulent friction. In such a case, the Euler equations possess a Bernoulli integral for the streamline:

$$
\begin{gathered}
B(r) \equiv \frac{1}{2} \rho\left(u^{2}+w^{2}+v^{2}\right)+\Delta p \ln u r=B\left(r_{1}\right), \\
p(r)=p_{1}+\Delta p \ln \frac{u r}{u_{1} r_{1}}, \quad \Delta p=p_{v} \equiv \rho \frac{u_{c}^{2}}{2}, \\
p_{1} \equiv p\left(r_{1}\right), \quad w=\frac{h}{r} \frac{\partial u r}{\partial r}, \quad v=\frac{a}{r} .
\end{gathered}
$$

Here $u, w$ and $v$ are the radial, vertical and tangential velocities, respectively, $r$ is distance from the center of the condensation area, $r=r_{1}$ is the outer border of the condensation area, $u_{1} \equiv u\left(r_{1}\right), \rho$ is air density, $u_{c}$ is the velocity scale determined by water vapor condensation, $a$ is angular momentum per unit air mass, and $z<h$ is the region of converging streamlines $(u>0)$.

It is convenient to use the following units

$$
\Delta p=1, \quad u_{c}=1, \quad \rho=2, \quad r_{1}=1 .
$$

In these units, the Bernoulli integral and the pressure potential become dimensionless

$$
\begin{aligned}
B(r)-B\left(r_{1}\right)= & u^{2}-u_{1}^{2}+w^{2}-w_{1}^{2} \\
& +\left(\frac{a^{2}}{r^{2}}-a^{2}+\ln \frac{u r}{u_{1}}\right)=0, \\
p(r)= & p_{1}+\ln \frac{u r}{u_{1}},
\end{aligned}
$$

where $w_{1} \equiv w\left(r_{1}\right)$ and $a \equiv v_{1} \equiv v\left(r_{1}\right)$.

Let us introduce a new variable $y \equiv u r / u_{1}$. Then Eq. (5) takes the form of a nonlinear differential equation on $y$ :

$$
\begin{gathered}
y^{\prime}=\frac{r}{u_{1} h} \sqrt{u_{1}^{2}\left(h^{2} y_{1}^{\prime 2}+1-\frac{y^{2}}{r^{2}}\right)-\left(\frac{a^{2}}{r^{2}}-a^{2}+\ln y\right)} \\
w \equiv u_{1} \frac{h}{r} y^{\prime}, \quad u \equiv u_{1} \frac{y}{r}, \quad v=\frac{a}{r}, \quad p=p_{1}+\ln y \\
y \equiv \frac{u r}{u_{1}}, \quad y^{\prime} \equiv \frac{d y}{d r}, \quad y_{1}^{\prime} \equiv y^{\prime}\left(r_{1}\right)
\end{gathered}
$$

Real solution of Eq. (7) exists at those $r$ only, where the expression under the square root in Eq. (7) is positive. The internal radius $r=r_{0}$, where condensation ceases, is obtained by equating the last term in the round brackets in Eqs. (5) and (7) to zero at $y\left(r_{0}\right)=r_{0}$, which is equivalent to $u\left(r_{0}\right)=u_{1}$. Condensation commences at $r_{1}$ and ceases at $r_{0}$ at one and the same radial velocity $u_{1}$. As follows from Eq. (7), the following relationships are simultaneously satisfied:

$$
\begin{gathered}
\frac{a^{2}}{r_{0}^{2}}-a^{2}+\ln r_{0}=0, \quad y_{0} \equiv y\left(r_{0}\right)=r_{0}, \\
y_{1} \equiv y\left(r_{1}\right)=1, \quad y_{0}^{\prime} \equiv y^{\prime}\left(r_{0}\right)=r_{0} y_{1}^{\prime}, \\
u_{0} \equiv u\left(r_{0}\right)=u_{1}, \quad w_{0} \equiv w\left(r_{0}\right)=w_{1}=u_{1} h y_{1}^{\prime} .
\end{gathered}
$$


At $r=r_{0}$ all condensational potential energy is converted to the kinetic energy of rotation. At $r<r_{0}$, real solutions of Eq. (7) for velocities $u, v$, and $w$ do not exist.

Equation (7) is a first-order differential equation with one boundary condition: $y_{1}=1\left(u\left(r_{1}\right)=u_{1}\right)$ or $y_{0}=r_{0}$ $\left(u\left(r_{0}\right)=u_{1}\right)$. If at fixed $y_{1}$ one considers the constant $y_{1}^{\prime}$ in Eq. (7) as a free parameter, then in the general case the interval, where real solutions exist, does not include the point $r=r_{0}$, which means that the maximum velocity $v_{\max } \sim a / r_{0}$ is not reached and the tornado does not exist.

Tornado exists, when the interval of real solutions comprises the point $r_{0}$ defined by Eq. (10). Solution of Eq. (7), that is real within the range $r_{0} \leqslant r \leqslant 1$, is obtained by setting the boundary condition on $y$ at $r_{0}$ as $y_{0}=r_{0}$ and choosing $y_{1}^{\prime}$ at given $u_{1}, a$ and $h$ such that $y_{1}=1$.

\section{COMPARISON WITH OBSERVATIONS}

Data of three-dimensional circulation (the dependencies of the velocities $u, w$ and $v$ on distance $r$ from the tornado center) have only recently become available and exist for three tornadoes [3-5]. We shall consider the Mulhall tornado (Oklahoma, 3 May 1999), which is the longest-lived (1 hour 20 $\min$ [6]) and longest-observed (18 min [5]) among the three as well as world's largest tornado on record [5].

According to empirical observations, the intense tornadoes can occur, when the mean relative humidity at $z \lesssim 1 \mathrm{~km}$ is not lower than $75-85 \%$ [7]. At a characteristic surface temperature $30{ }^{\circ} \mathrm{C}[8]$ and $80 \%$ relative humidity the vapor pressure at the surface is $p_{v}=\Delta p \simeq 30 \mathrm{hPa}$. Taking air density $\rho=1.15 \mathrm{~kg} \mathrm{~m}^{-3}$ in Eq. (2), we obtain the characteristic velocity $u_{c}=73 \mathrm{~m} \mathrm{~s}^{-1}$. Velocities $v_{1}$ and $u_{1}$ at the external border $r=r_{1}$ must be the functions of translational velocity $U$ (speed of movement of tornado as a whole). We put radial velocity $u_{1}=U / \pi$ [2], taking into account that the flux of moist air via tornado cross-section $2 r_{1} U$ is equal to the flux via tornado circumference $2 \pi r_{1} u_{1}$. We put tangential velocity $v_{1}=2 U / \pi$ assuming that the angular momentum of the main streamline that delivers moist air into the condensation area (see, e.g., Fig. 8 in work [9]) is determined by the mean value of $U \cos \alpha$. Here $0 \leqslant \alpha \leqslant \pi / 2$ is a random angle between velocity at this streamline and radius-vector $r$ at the point $r=r_{1}$, where the air enters the condensation area. From $U=13 \mathrm{~m} \mathrm{~s}^{-1}$ [5] we have for dimensionless variables $u_{1}=U / \pi u_{c}=0.06, v_{1}=2 U / \pi u_{c}=0.12$. For $a=v_{1}=0.12$, we obtain the eye radius $r_{0}=0.074$ from Eq. (10). Taking cloud height $h=1.2 \mathrm{~km}$ and total size of tornado condensation area $r_{1}=8.5 \mathrm{~km}$, we have dimensionless value $h=0.14$.

For these particular parameters the numerical solution of Eq. (7) obtained by using conditions (10) and (11) corresponds to $y_{1}^{\prime}=0.03574$ (see Fig. 11A). The account of stationary eye rotation is made in the same way as in work [2], when a certain part of tangential kinetic energy developed in the condensation area is spent on solid-body rotation and creation of the pressure gradient in the eye of radius $r_{0}$. This lowers tangential velocity in the transitional region $r_{0} \leqslant r<r_{e}$ between the condensation area and the eye, where $r_{e}=1.65 r_{0}$ [2]. The expressions for tangential velocity and pressure at $r<r_{0}$ coincide with Eqs. (23)-(25) in work [2]. The empirical points shown in Fig. 11B characterize the Mulhall tornado close to the time of peak intensity. They correspond to characteristic vertical velocity $w(r)$ at $z=650 \mathrm{~m}$ [5, Fig. 4b], mean radial velocity $u(r)$ at $150 \mathrm{~m}<z<850 \mathrm{~m}[5$, Fig. 4b] and mean tangential velocity $v(r)$ at $50 \mathrm{~m}<z<950 \mathrm{~m} \mathrm{[5}$, Fig. 5a].

It is seen from Fig. 13 that to the right side of the maximum the radial distribution of mean tangential velocity at $z \leqslant h$ conforms well to the assumption of conserved angular momentum (3). The choice of $h=1.2 \mathrm{~km}$ is supported by observation that in this layer the radial velocity $u(r)$ exceeds $u_{1}=4.4 \mathrm{~m} \mathrm{~s}^{-1}$ over considerable part of tornado circulation [5, Fig. 4b]. (It should be noted that two other tornadoes, for which the data are available [3, 4], have a significantly lower inflow level, $h \leqslant 400 \mathrm{~m}$, than the Mulhall tornado. Mean tangential velocities do not follow the conserved angular momentum distribution. The decrease of angular momentum towards the center demands a more detailed consideration with additional parameters [2]).

Total pressure fall as shown in Fig. 11A is $3.6 \Delta p=108 \mathrm{hPa}$. This is in agreement with the few available direct measurements of tornado surface pressure. In the Manchester tornado (South Dacota, 2003), which was of the same (F4) intensity as the Mulhall tornado, a pressure fall of $100 \mathrm{hPa}$ was registered [5].

\section{CONDITIONS OF VORTEX EXISTENCE AND THE MAXIMUM POSSIBLE VELOCITIES}

With account of stationary eye rotation [2] the maximum wind velocity $v_{\max }=a / r_{e}$ (and, correspondingly, the maximum kinetic energy) is achieved at $r_{e}=1.65 r_{0}$ (see Fig. 11A), where $r_{0}$ is a function of $a$ given by Eq. (10), Fig. 2A. The Earth rotation does not determine angular momentum in tornado due to the small linear size of the vortex. The value of $a$ is related to the translational velocity $U$. This velocity cannot be infinitely small: tornado exists at the expense of water vapor accumulated in the atmosphere and, hence, must move to sustain itself [10, pp. 227-229]. Maximum velocity attainable in the condensational vortex depends only weakly on angular momentum and grows rather slowly (logarithmically) with decreasing $a$ (see Fig. 22A). For realistic $a \geqslant 10^{-3}$ $\left(v_{1} \geqslant 0.1 \mathrm{~m} \mathrm{~s}^{-1}\right), v_{\max }$ does not exceed $1.7 u_{c} \simeq 120 \mathrm{~m} \mathrm{~s}^{-1}$. This agrees well with the available estimates of maximum wind speeds in tornadoes [5].

The existence of vortex is related to a certain minimum value of radial velocity $u$, which describes the atmospheric inertia with respect to the development of condensational cir- 

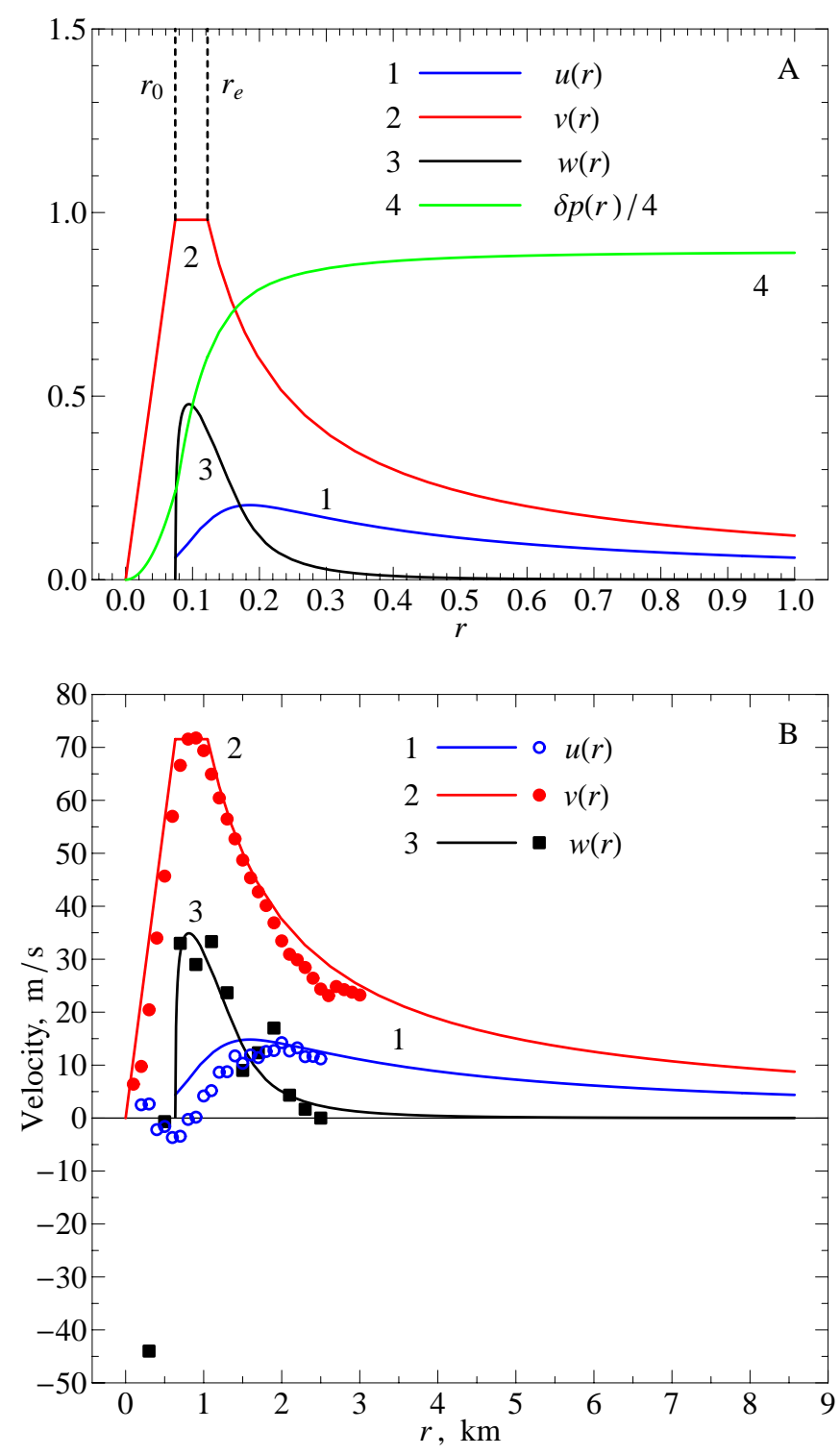

FIG. 1: (color online). A: Solution of Eq. (7) at $a=0.12$ $\left(r_{0}=0.074\right), u_{1}=0.06, h=0.14$ in dimensionless units (4), $\delta p(r) \equiv p(r)-p(0)$. B: Comparison with observations for the Mulhall tornado [5] at $u_{c}=73 \mathrm{~m} \mathrm{~s}^{-1}, h=1.2 \mathrm{~km}$ $\left(r_{1}=8.5 \mathrm{~km}\right)$. The negative vertical velocity (downdraft) within the tornado eye and the decrease of radial velocity near $r_{0}$ is related to non-stationarity of eye rotation not described by the Bernoulli integral (7), the latter pertaining to the converging ascending streamline.

culation. At $u<u_{1}$ condensation ceases. The condition $u_{0}=u_{1}$ corresponds to the following relationships

$$
\begin{aligned}
& u^{\prime}\left(r_{0}\right)=\frac{u_{0}}{r_{0}}\left(y_{0}^{\prime}-1\right)=u_{1}\left(y_{1}^{\prime}-\frac{1}{r_{0}}\right) \simeq-\frac{u_{1}}{r_{0}}, \\
& u^{\prime}\left(r_{1}\right)=u_{0}\left(\frac{y_{0}^{\prime}}{r_{0}}-1\right)=u_{1}\left(y_{1}^{\prime}-1\right) .
\end{aligned}
$$

This means that there is a minimum of $u(r)$ at $r=r_{\min }$
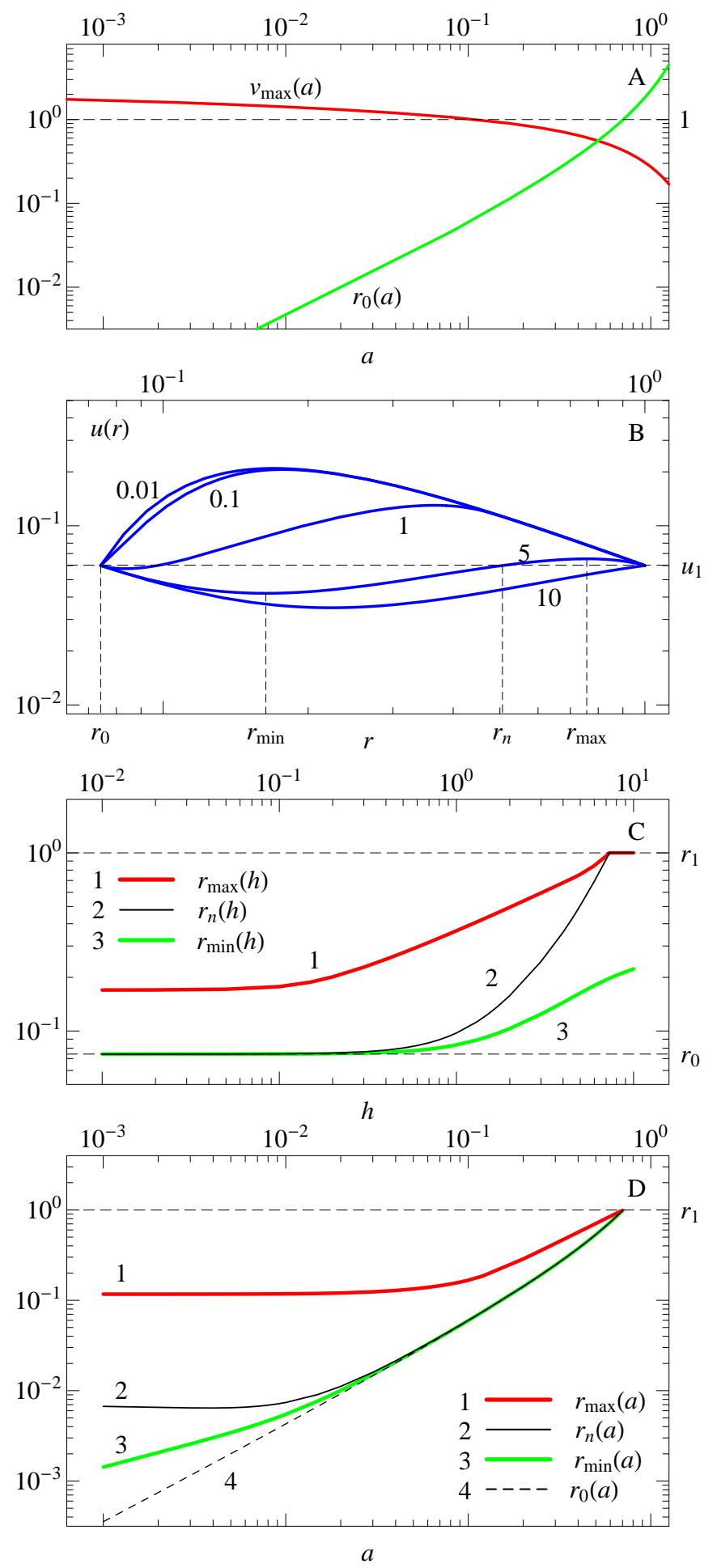

FIG. 2: (color online). A: Dependence of eye radius $r_{0}$ and maximal tangential velocity $v_{\max }=a / r_{e}, r_{e}=1.65 r_{0}$ [2] on angular momentum $a$ in dimensionless units (4). B: Radial velocity $u(r)$ obtained by solving Eq. (7) under conditions

(10) and (11) at $a=0.12, u_{1}=0.06$ for five values of $h$ (shown near the curves). Points $r_{\min }, r_{\max }$ and $r_{n}$ shown for $h=5$ correspond to radial velocity minimum, maximum and $u\left(r_{n}\right)=u_{1}$, respectively. C: Dependence of $r_{\min }, r_{\max }$ and $r_{n}$ on $h$ at $a=0.12, u_{1}=0.06$. D: Dependence of $r_{\min }$,

$r_{\max }$ and $r_{n}$ on $a$ at $h=0.14, u_{1}=0.06 ; r_{0}(a)$ 10). 
within $r_{0}<r_{\min }<r_{1}$. The existence of condensation at $u(r)>u_{1}$ means that there is also a point $r=r_{\max }$, $r_{0}<r_{\max }<r_{1}$, where $u(r)$ is maximum. It follows that there is a point $r=r_{n}, r_{0}<r_{\min }<r_{n}<r_{\max }<r_{1}$, where $u\left(r_{n}\right)=u_{1}$. At $r<r_{n}$ there is no condensation and no condensational pressure potential to accelerate air. When $r_{n} \gg r_{0}$ the maximum vortex velocity, $a / r_{e}$, is not reached: $v_{\max }=a / r_{n} \ll a / r_{e}$. Therefore, tornado exists, if the following condition $\kappa \equiv\left(r_{n}-r_{0}\right) / r_{0} \ll 1$ is fulfilled $\left(\kappa \simeq 10^{-3}\right.$ for the vortex shown in Fig. 1.

Analysis of Eq. (7) shows that this condition is violated with increasing $h$, which, at a fixed height of the atmosphere, corresponds to diminishing linear size $r_{1}$ of the condensation area. In Fig. 2B, profiles of $u(r)$ are shown for $h$ varying from 0.01 to 10 . At $h \geqslant 7.3$ we have $y_{1}^{\prime} \geqslant 1, u^{\prime}\left(r_{1}\right) \geqslant 0$ and maximum of $u(r)$ at $r<r_{1}$ disappears. Decreasing $a$ at fixed $h$ also leads to increasing $\kappa$, Fig. $2 \mathrm{D}$. It follows that the smaller the horizontal size of the condensation area, the larger the angular momentum that is needed for a vortex to arise. A given value of angular momentum sets the minimal horizontal size of the vortex. For the parameters shown in Fig. 1 the minimum possible vortex, where velocity $v \simeq u_{c} \simeq 70 \mathrm{~m} \mathrm{~s}^{-1}$ can be observed, corresponds to $h \sim 1$ (see Fig. 2 C). The minimal condensation area has then radius $r_{1} \sim 1.2 \mathrm{~km}$ and funnel (eye) radius of about $90 \mathrm{~m}$. At small $a$ and $r_{1}$ only ordinary squalls can develop.

* Corresponding author: anef@ thd.pnpi.spb.ru

[1] A. M. Makarieva and V. G. Gorshkov, Phys. Lett. A 373, 4201 (2009).

[2] A. M. Makarieva and V. G. Gorshkov, Phys. Lett. A 375, 1053 (2011).

[3] K. Kosiba and J. Wurman, J. Atmos. Sci. 67, 3074 (2010).

[4] K. A. Kosiba, R. J. Trapp, and J. Wurman, Geophys. Res. Lett. 35, L05805 (2008).

[5] W.-C. Lee and J. Wurman, J. Atmos. Sci. 62, 2373 (2005).

[6] D. A. Speheger, C. A. Doswell, and G. J. Stumpf, Wea. Forecast. 17, 362 (2002).

[7] R. L. Thompson, R. Edwards, J. A. Hart, K. L. Elmore, and P. Markowski, Wea. Forecast. 18, 1243 (2003).

[8] R. L. Tanamachi, H. B. Bluestein, S. S. Moore, and R. P. Madding, J. Atmos. Oceanic Technol. 23, 1445 (2006).

[9] J. Wurman, K. Kosiba, P. Markowski, Y. Richardson, D. Dowell, and P. Robinson, Mon. Wea. Rev. 138, 4439 (2010).

[10] V. G. Gorshkov, Physical and Biological Bases of Life Stability (Springer, Berlin, 1995). 